\title{
Development of Scanning Stress Measurement Method Using Laser Photoelasticity*
}

\author{
Yasushi NIITSU**, Kenji GOMI** \\ and Kensuke ICHINOSE**
}

\begin{abstract}
We have developed an optical equipment that possesses high detection sensitivity for measuring the small optical retardation induced by small stress by means of laser photoelasticity. A He- $\mathrm{Ne}$ laser is used as a light source to measure small stress in transparent materials. We explain the theory and process of the measurement of optical retardation in the materials. The magnitudes of principal stress difference and the directions of the principal stress are obtained simultaneously and quantitatively using our equipment. To evaluate the validity of the measurement results of the equipment, the stress distribution of a pulled rectangular glass plate with notches at both sides is measured using the equipment. The experimental results of stress distribution agree well with the analytical results of FEM. The stress distribution can be determined quickly by using the equipment and scanning stress distribution measurement has been realized.
\end{abstract}

Key Words: Material Testing, Experimental Stress Analysis, Nondestructive Inspection, Finite Element Method, Photoelastic Modulator, Laser Photoelasticity

\section{Introduction}

Recently, the production technology of small precision devices, for example, VLSIs and sensor devices, has been significantly advanced. These devices have been widely employed in mechanical and electrical apparatus. They consist of many components bonded or molded with resins. These complex systems sometimes break due to thermal stress induced by a mismatch of thermal expansions. Therefore, the development of evaluation technology for stress states in electrical devices is required in various fields. Several techniques have been developed to evaluate the stress states in complex small devices, for example, laser Raman microspectroscopy and ultrasonic microscopy. On the other hand, an optical stress analysis method like photoelasticity is useful for the microscopic stress distribution evaluation.

* Received 20th February, 1997. Japanese original: Trans. Jpn. Soc. Mech. Eng., Vol. 62, No. 600, A (1996), pp. 1883-1888 (Received 19th February, 1996)

** Department of Mechanical Engineering, Tokyo Denki Univ., 2-2 Kanda-Nishikicho, Chiyodaku, Tokyo 101, Japan
Jasperson and Schnattely ${ }^{(1)}$ found that the polarization modulation is applicable for the measurement of the optical anisotropy, and Modine, Major and Sonder ${ }^{(2)}$ studied the optical birefringence using the photoelastic modulation. Yamada ${ }^{(3)}$ applied the polarization modulation to the stress analysis. However, that method is not suitable for high-speed measurement.

This paper presents a new stress measuring method and equipment that are suitable for the highspeed measurement. The equipment is a kind of circular polariscope using a photoelastic modulator and polarized laser ${ }^{(4)-(8)}$. In order to evaluate the validity of the measurement results of this equipment, the stress distribution of a pulled rectangular glass plate with notches at both sides is measured by using the equipment. The stress distribution corresponding to the optical birefringence distribution was compared with the results of Finite Element Method analysis.

\section{Measuring Equipment and Theory}

\subsection{Birefringence measurement equipment}

Figure 1 shows the construction of the birefringence measuring equipment. This equipment can 


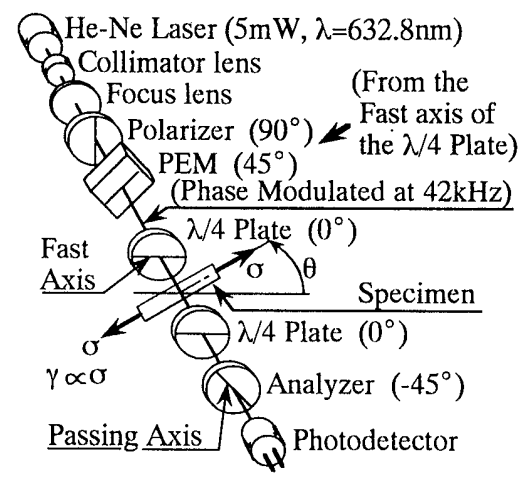

Fig. 1 Schematic figure of birefringence measurement system

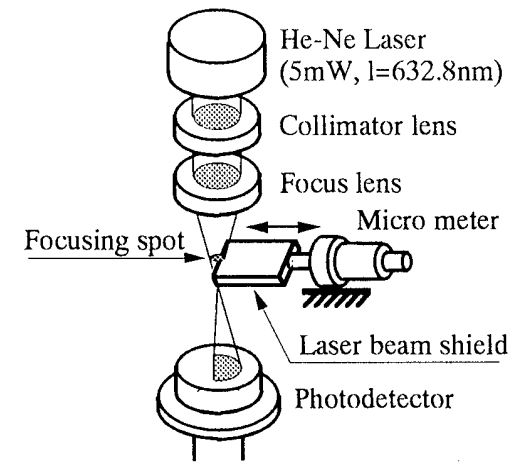

(a) Measuement method

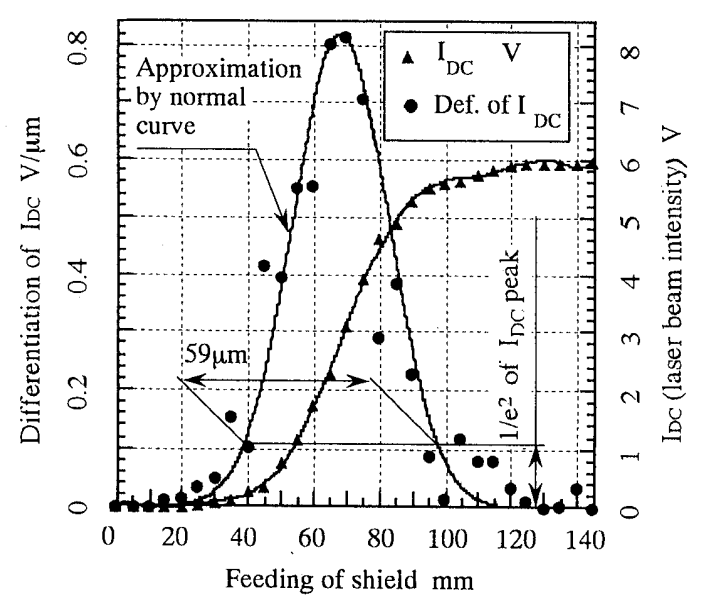

(b) The relation between shield displacement and laser beam intensity

Fig. 2 The measurement of laser beam diameter on specimen

measure the birefringence without a specimen rotation. A $5 \mathrm{~mW} \mathrm{He}-\mathrm{Ne}$ laser light $(\lambda=632.8 \mathrm{~nm})$ passes through a collimator lens, a focus lens, a polarizer(Glan-Thompson Prism), a PEM(Photo Elastic Modulator), a quarter wave plate, a birefringent specimen (a stressed specimen), a quarter wave plate and analyzer. After passing the analyzer, the laser light is detected by a photodetector. This PEM converts linearly polarized light into oscillating ellip- tic light at its $42 \mathrm{kHz}$ resonant frequency. The direction of the birefringence axis of the PEM inclines $90^{\circ}$ from the axis of the analyzer, and $45^{\circ}$ from the axis of the polarizer. The directions of the fast axes of the quarter wave plates incline $45^{\circ}$ from the axis of the analyzer, and $90^{\circ}$ from the axis of the polarizer.

The laser light is concentrated upon the transparent specimen by focus lens. The spot of the laser light is a round shape. The diameter of the laser spot on the specimen is measured as shown in Fig. 2(a). A light intensity $I_{D C}$ is measured by the photodetector. Figure 2(b) shows the relation between $I_{D C}$ and the displacement of the beam shield plate. The horizontal axis shows the displacement of the shield. The right vertical axis shows $I_{D C}$. The points of the triangle marks in Fig. 2(b) show the relation between $I_{D C}$ and the shield displacement. On the other hand, the round marks show the differentiation of $I_{D C}$ with respect to the displacement of the shield, and the left vertical axis shows it. The differentiation is approximated by the normal curve shown in Fig. 2(b), because the beam of the laser light is Gaussian Beam. Since such a beam is circular in cross $^{-}$section, the diameter at which the light intensity is $1 / e^{2}$ of its value on the axis is called beam width. The full width of the normal curve at auxiliary line corresponds to the beam width and the diameter of the laser spot is about $59 \mu \mathrm{m}$. The average retardation and birefringence direction in the area of this beam width can be obtained numerically by analyzing the intensity of the light through the analyzer.

\subsection{Relation between stress and transmitted light intensity}

Jones'calculus computation gives the light intensity $I$ that comes through the analyzer as follows,

$$
I=\frac{\alpha I_{0}}{4}\{1+\sin \gamma(\cos \delta \cdot \sin 2 \theta-\sin \delta \cdot \cos 2 \theta)\}
$$

where $\alpha$ is the transparency of the specimen, $I_{0}$ is the light intensity before transmitting by the polarizer, $\theta$ is the angle between the birefringence axis of the specimen and the fast axes of the quarter wave plates as shown in Fig. 1. If the specimen is the isotropic material, the birefringence direction of the specimen coincides with the principal stress direction. The notations $\gamma$ and $\delta$ show the retardations of the specimen and PEM, respectively. If the specimen is optically isotropic, the retardation $\gamma$ is proportional to the principal stress difference $\left(\sigma_{1}-\sigma_{2}\right)$ according to Brewster's law. Therefore, the principal stress difference and its direction can be obtained by calculating $\gamma$ and $\theta$ in Eq. (1). The retardation $\delta$ of the PEM can be expressed as sinusoidal change of $42 \mathrm{kHz}$ resonant frequency. These retardations are written as 
follows,

$$
\begin{aligned}
& \gamma=2 \pi C\left(\sigma_{1}-\sigma_{2}\right) \frac{d}{\lambda} \\
& \delta=\delta_{0} \cdot \sin \omega t
\end{aligned}
$$

where $C$ is the stress-optical coefficient(Brewster's constant). The notation $d$ is the thickness of the specimen (the pass way length of the laser light in the specimen), $\lambda$ the wavelength of the $\mathrm{He}^{-\mathrm{Ne}}$ laser light $(632.8 \mathrm{~nm}), \delta_{0}$ the amplitude of the modulator retardation that is proportional to the applied oscillating PEM voltage, and $\omega$ is the resonant angular frequency of the PEM. Substituting Eq. (3) into Eq. (1) and rearranging, the following Fourier expansion is obtained,

$$
\begin{aligned}
& I=I_{D C}+I_{A C 1} \sin \omega t+I_{A C 2} \cos 2 \omega t \\
& \quad+I_{A C 3} \sin 3 \omega t+\cdots \\
& I_{D C}=\frac{\alpha I_{0}}{4}\left\{1+J_{0}\left(\delta_{0}\right) \cdot \sin \gamma \cdot \sin 2 \theta\right\} \\
& I_{A C 1}=-\frac{\alpha I_{0}}{2} J_{1}\left(\delta_{0}\right) \cdot \sin \gamma \cdot \cos 2 \theta \\
& I_{A C 2}=\frac{\alpha I_{0}}{2} J_{2}\left(\delta_{0}\right) \cdot \sin \gamma \cdot \sin 2 \theta
\end{aligned}
$$

where $I_{A C 1}$ and $I_{A C 2}$ are the alternating light intensities of resonance frequency and its double frequency, $I_{D C}$ is the direct component of the light intensity. Both $I_{A C}$ 1 and $I_{A C 2}$ are measured by lock-in amplifiers. The direct component $I_{D C}$ is measured by a voltmeter. $J_{0}\left(\delta_{0}\right) \sim J_{2}\left(\delta_{0}\right)$ are Bessel functions. The second term of Eq. ( 5 ) can be eliminated by adjusting the PEM voltage, so that $I_{D C}$ becomes constant,

$$
I_{D C}=\frac{\alpha I_{0}}{4}
$$

The direct component $I_{D C}$ is independent of both $\theta$ and $\gamma$ of the specimen. Dividing Eq. ( 6 ) and ( 7 ) by Eq. (8), we can obtain,

$$
\begin{aligned}
& \frac{I_{A C 1}}{I_{D C}}=A_{1} \cdot \sin \gamma \cdot \cos 2 \theta \\
& \frac{I_{A C 2}}{I_{D C}}=A_{2} \cdot \sin \gamma \cdot \sin 2 \theta
\end{aligned}
$$

where, $A_{1}$ and $A_{2}$ are constants and independent of both the transparency and stress state of the specimen. They are determined with the experimental results of quarter wave plate as a specimen. That detail is mentioned later. The values of $\gamma$ and $\theta$ of the specimen are obtained from Eq. (9) and (10),

$$
\begin{aligned}
& \gamma=\sin ^{-1}\left[\sqrt{\left(\frac{I_{A C 1}}{I_{D C} \cdot A_{1}}\right)^{2}+\left(\frac{I_{A C 2}}{I_{D C} \cdot A_{2}}\right)^{2}}\right] \\
& \theta=\frac{1}{2} \tan ^{-1}\left[\frac{A_{1} \cdot I_{A C 2}}{A_{2} \cdot I_{A C 1}}\right]
\end{aligned}
$$

where $\gamma$ is absolute value of $\gamma$ in Eq. (2). If the photoelastic constant $C$ of a specimen is positive, the first principal stress direction corresponds with the birefringence direction $\theta$. On the other hand, if the constant $C$ is negative, the second principal stress direction corresponds with $\theta$.
The range of $\theta$ in Eq. (12) is only between $-\pi / 4$ and $\pi / 4$. The signs of $I_{A C 1}$ and $I_{A C 2}$ are necessary to determine the actual first principal stress direction $\theta$. The signs of $I_{A C 1}$ and $I_{A C 2}$ are obtained from the phase difference between the applied oscillating PEM voltage and the detected voltage of photodetector ${ }^{(5)}$. If the phase difference between the PEM voltage and $I_{A C 1}$ is in phase, the sign of $I_{A C 1}$ is positive. If that is antiphase, $I_{A C 1}$ is negative. The sign of $I_{A C 2}$ can be obtained from the relation between the $2 \omega$ phase and $I_{A C 2}$ phase, similarly. These phase states such as inphase or antiphase can be obtained by lock-in amplifiers. Figure 3 shows the relation between the signs of $I_{A C 1}, I_{A C 2}$ and the first principal stress direction $\theta$. The horizontal axis in Fig. 3 coincides with the fast axis of the quarter wave plate.

\subsection{Measurement of quarter wave plate retardation}

To determine the constants $A_{1}$ and $A_{2}$ in Eq. (9) and (10), the optical retardation of quarter wave plate whose retardation is accurately known is measured. When the quarter wave plate is adopted as the sample of measurement, the maximum values of $I_{A C 1}$ and $I_{A C 2}$ are obtained because of the retardation of quarter wave plate $\gamma=\pi / 2$. In this case, the amplitudes of $\left(I_{A C 1} / I_{D C}\right)$ and $\left(I_{A C 2} / I_{D C}\right)$ are equal to $A_{1}$ and $A_{2}$, respectively, and we obtain $A_{1}=-0.0682$ and $A_{2}=$ 0.0178. Figure 4 shows the trajectory of $\left(I_{A C 1} /\right.$ $\left.\left(I_{D C} \cdot A_{1}\right)\right)$ and $\left(I_{A C 2} /\left(I_{D C} \cdot A_{2}\right)\right)$ of the quarter wave plate during rotating. The radius and argument of the measured point in this coordinate correspond to the magnitude and the direction of birefringence according to Eq. (11) and (12).

\subsection{Measurement of stress-optical coefficient of the glass}

The stress distribution in the glass plate (super frost white: commercially available) has been measured to examine the measurement validity of the equipment. Glass is isotropic material and has low

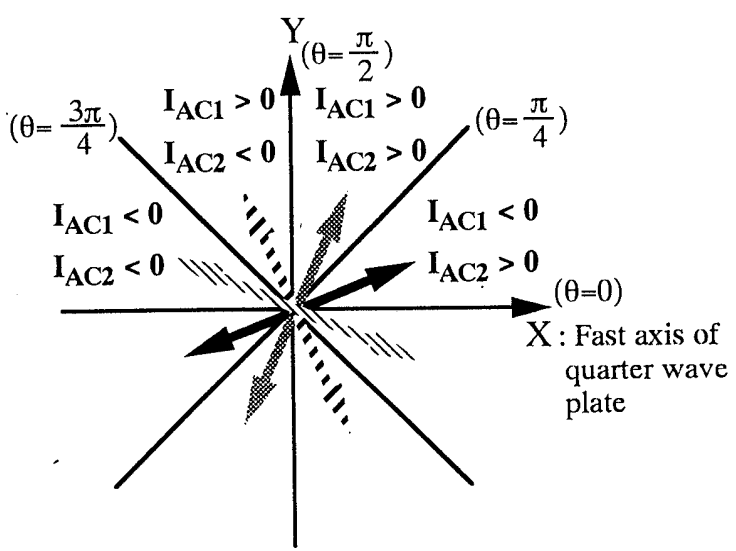

Fig. 3 The relation between the signs of $I_{A C}$ and the first principal stress direction, $\theta$ 


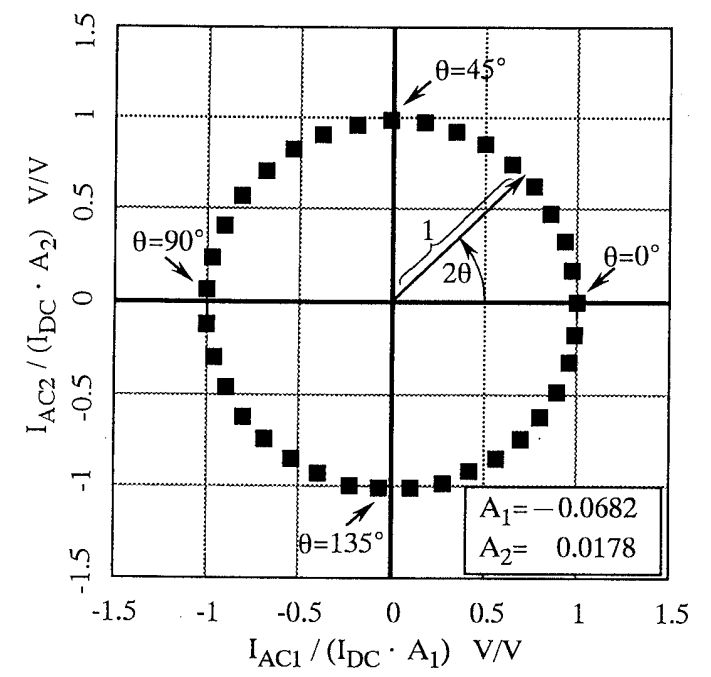

Fig. 4 The trajectory of $I_{A C 1} /\left(I_{D C} \cdot A_{1}\right)$ and $I_{A C 2} /\left(I_{D C} \cdot A_{2}\right)$ of quarter wave plate

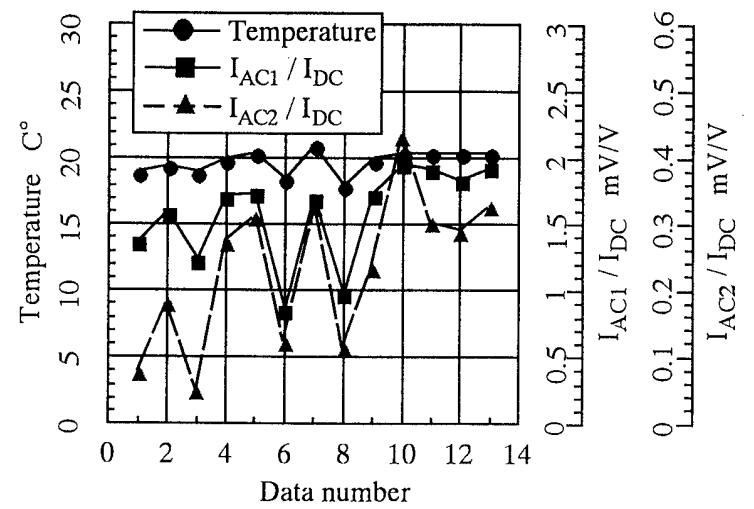

Fig. 6 The relation between temperature and $I_{A C} / I_{D C}$ without sample

stress-optical coefficient. The coefficient of glass is about one sixteenth of epoxy. The glass specimen is suitable for the stress measurement.

The relation between the stress and the measurement value of the glass plate is investigated. Figure 5 (a) shows the tensile specimen whose size is 40.4 $\mathrm{mm}$ long, $5.15 \mathrm{~mm}$ wide and $0.993 \mathrm{~mm}$ thick. The specimen is reinforced with PMMA plates at both ends. The tensile load is applied to longitudinal direction with a screw type loading equipment. The birefringence of the specimen is measured by our equipment at every $15^{\circ}$ during rotating the specimen from $0^{\circ}$ to $180^{\circ}$; the rotating axis coincides with the optical axis. The experimental results of $\left.\left(I_{A C 1} / I_{D C} \cdot A_{1}\right)\right)$ and $\left.\left(I_{A C 2} / I_{D C} \cdot A_{2}\right)\right)$ are shown in Fig. 5 (b). Equations ( 9 ) and (10) formulate the circle; the parameter is $2 \theta$. Therefore, all the circles ought to be concentric circles whose centers coincide with the origin. However, all of them are not concentric, and the centers do not coincide with the origin. Figure $5(\mathrm{c})$ is obtained by making a parallel translation of

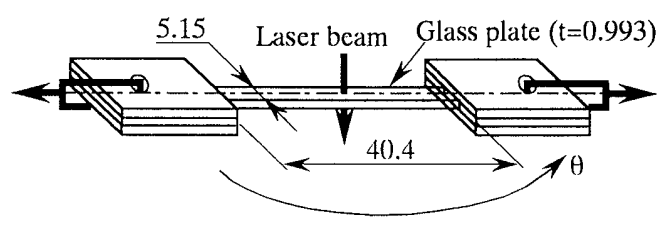

(a) The glass specimen

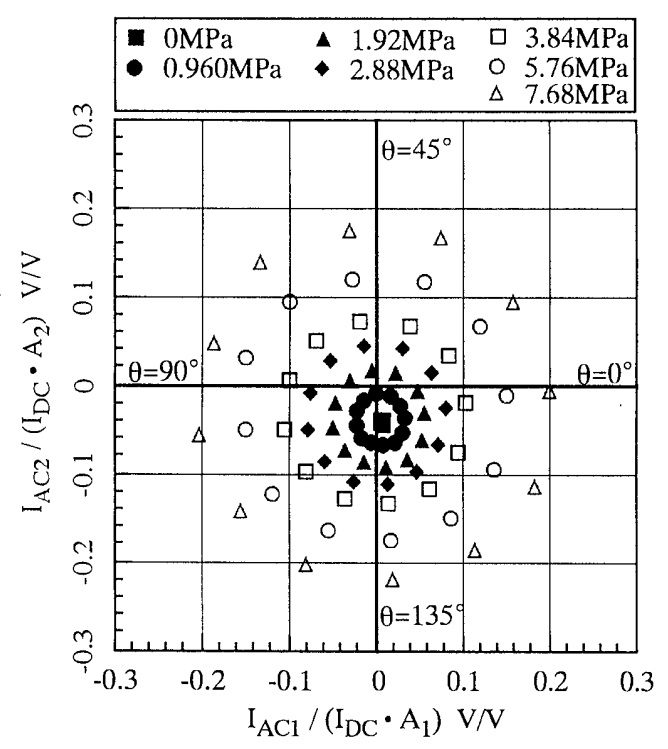

(b) before corrections

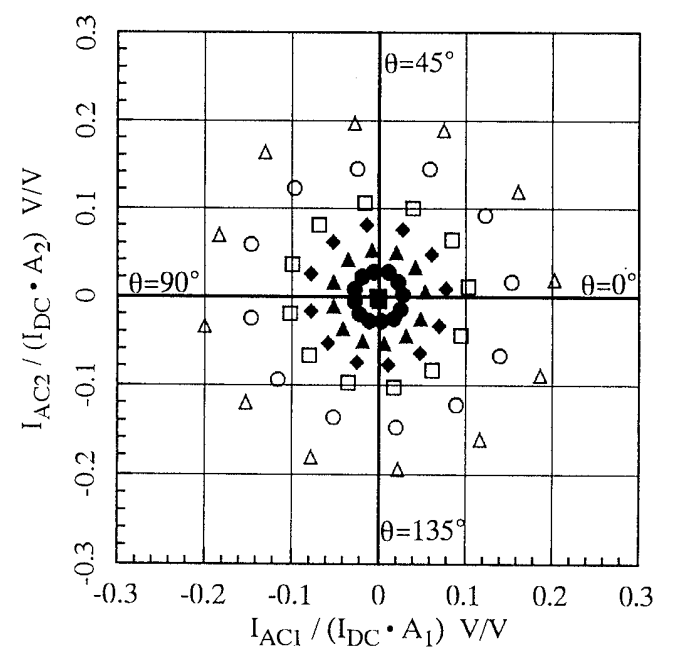

(c) after corrections

Fig. 5 The glass specimen and the trajectory of $I_{A C 1} /\left(I_{D C} \cdot a\right)$ and $I_{A C 2} /\left(I_{D C} \cdot b\right)$ of the specimen; (a): the glass specimen, (b): before corrections, (c) : after corrections

each circle in Fig. 5(b). It is seemed that temperature influences the shifts of the experimental results in Fig. 5 (b). Figure 6 shows the relation between room temperature and the measured values of $\left(\left(I_{A C 1} / I_{D C}\right)\right.$, $\left.\left(I_{A C 2} / I_{D C}\right)\right)$ with no specimen. The horizontal axis shows the time. The left-hand and right-hand vertical axes show the temperature, $\left(I_{A C_{1}} / I_{D C}\right)$ and $\left(I_{A C 2} / I_{D C}\right)$, respectively. The values of $\left(\left(I_{A C 1} / I_{D C}\right)\right.$ and 
$\left.\left(I_{A C 2} / I_{D C}\right)\right)$ should be as small as possible. From Data6 and 8 in Fig. 6 , the shift of origin of the measurement becomes smallest at $18.5^{\circ} \mathrm{C}$. Therefore, the accurate control of room temperature can inhibit the shifts in the experimental results shown in Fig. 5(b). It is observed that the coordinates of the points in Fig. 5 (c) correspond with the optical retardation and its birefringence direction. Since glass is optically isotropic and its photoelastic constant is positive, the optical retardation $\gamma$ is proportional to the principal stress difference $\left(\sigma_{1}-\sigma_{2}\right)$ and the birefringence direction $\theta$ corresponds with the first principal stress direction. The difference between $\theta$ and the first principal stress direction in this experiment is less than $3.41^{\circ}$. Figure 7 shows the relation between the optical retardation $(\gamma \lambda / 2 \pi[\mathrm{nm}])$ and the load per unit width $(\sigma \cdot d[\mathrm{MPa} \cdot \mathrm{mm}])$. That relation is obtained from the experimental results in Fig. 5(c). Each point in Fig. 7 is the average of the twelve measurements. Their error bars show standard devia-

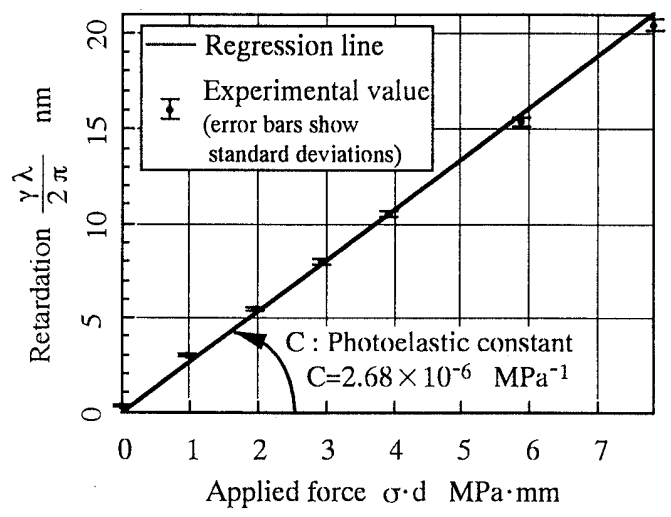

Fig. 7 The relation between retardation and applied force per unit width

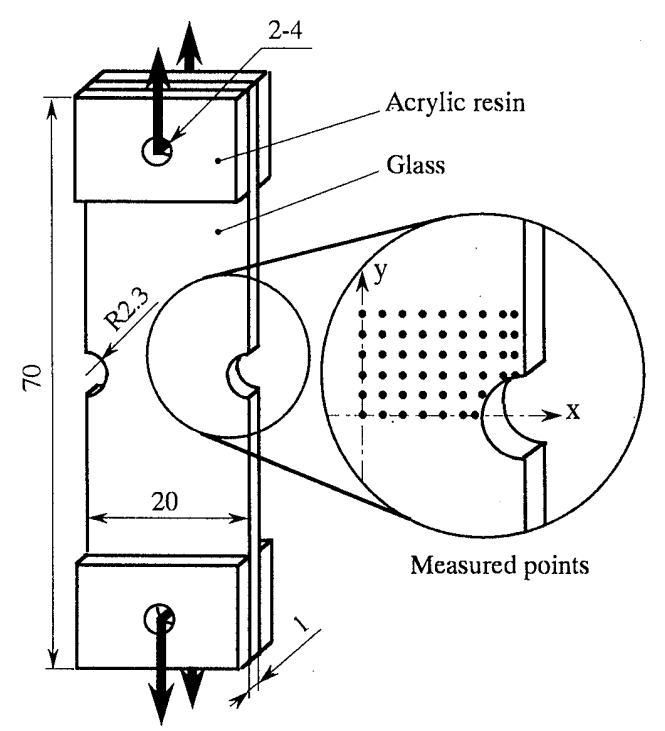

Fig. 8 The shape and stress measurement points of the glass plate specimen with notches at its both sides tions. A line shows the regression line through the origin. According to Eq. (2), the inclination of the line is the stress ${ }^{-}$optical coefficient of the glass used in the experiment : $C=2.68 \times 10^{-6}[1 / \mathrm{MPa}]$.

\section{Measurement of stress distribution and discussion}

In order to evaluate the validity of the measurement results of our birefringence measuring equipment, the measured stress distribution of a pulled rectangular glass plate with notches at both sides is compared with the calculation results of FEM. Figure 8 shows the dimension of the notch specimen and the points of measured in it. The rectangular coordinate system in Fig. 8 has the origin on the center of the specimen and its $y$ axis is parallel with the loading direction. Two steel pins of $3 \mathrm{~mm}$ diameter are inserted into the holes of the specimen and the tensile load of $60 \mathrm{~N}$ is applied through the pins. The measurement is carried out at $1 \mathrm{~mm}$ interval in both $x$ and $y$ directions and at the point of $0.3 \mathrm{~mm}$ inside from the boundary. Figure 9 shows the mapping of node-points on the specimen and the boundary conditions used in FEM analysis. A display III is used for the pre-postprocessor, and NISA II is used for the solver for the analysis.

Figure 10 shows the comparison of the experimental results with the results of FEM analysis; (a) is the experimental result and ( $b$ ) is the FEM result. The length and direction of arrows show the principal stress difference and direction of the first principal stress, respectively. It is observed that the states of stress concentration near the semicircular notch and the tendencies of the first principal stress directions are measured well. The experimental results near the

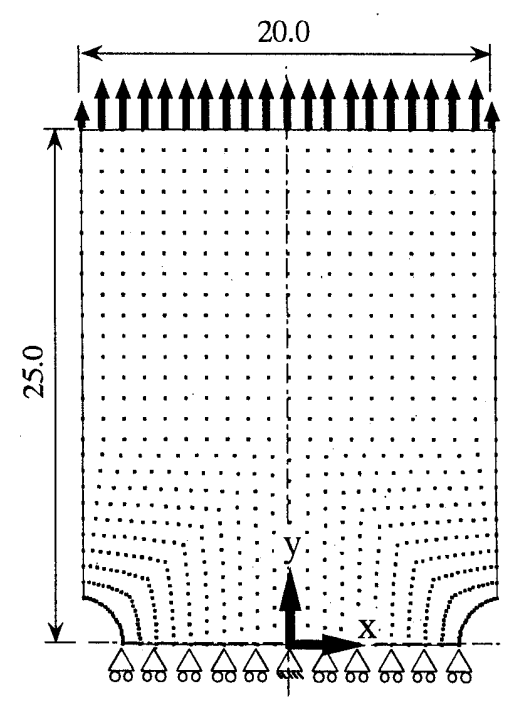

Fig. 9 The map of node-points on the specimen and boundary conditions used in FEM analysis 


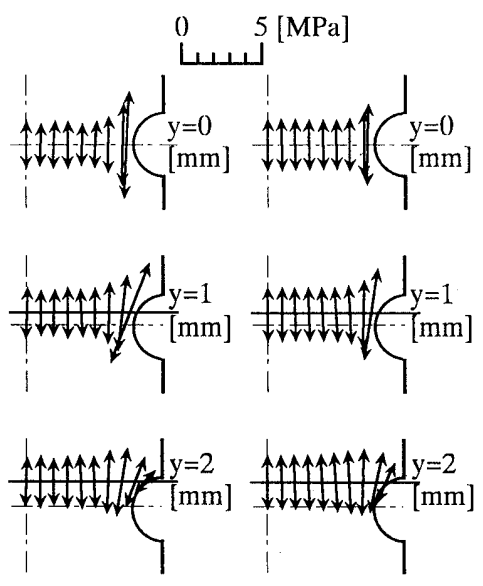

(a) Experiment results

(b) FEM analysis

Fig. 10 Comparison of experimental results with FEM analysis; (a ): experimental results, (b): FEM analysis

semicircular notch are about 20\% larger than FEM results, and in the other area, the experimental results are about $10 \%$ smaller than FEM results.

\section{Conclusion}

We have developed an optical equipment that possesses high detection sensitivity for measuring small optical retardation induced by stress. The equipment can measure the retardation of the specimen without rotating. Therefore, the equipment needs less than 0.1 seconds during measuring the retardation at a point. The stress distribution corresponding to optical retardation distribution is compared with the results of FEM analysis. It is confirmed that the states of stress concentration near the semicircular notch and the tendencies of the first principal stress directions are measured well. The features of the optical equipment and future applications are as follows.

(1) This optical equipment can be applicable to the dynamic stress evaluation like thermal stress distribution change.

(2) This method can achieve higher spacial-reso- lution up to 1 micrometer with the lens system like a microscope.

(3) It is possible to evaluate the stress states in engineering plastics, ceramics and semiconductor wafers by adopting the laser of the appropriate wavelength as a light source, for example infrared laser.

\section{Acknowledgements}

This study is partly supported by the Scientific Research Grant from Japanese Ministry of Education.

\section{References}

(1) Jasperson, S. N. and Schnatterly, S. E., An Improved Method for High refractivity Ellipsometry Based on a New Polarization Technique, Rev. Sci. Instrum., Vol. 40, No. 6(1969), p. 761.

(2) Modine, F. A., Major, R. W. and Sonder, E., High Frequency Polarization Method for Measuring Birefringence, J. Appl. Opt., Vol. 14, No. 3(1975), p. 757.

(3) Yamada, M., High-sensitivity computercontrolled infrared polariscope, Rev. Sci. Instrum., Vol. 64, No. 7(1993), p. 1815.

(4) Ichinose, K., Niitsu, Y. and Ikegami, K., Residual Stress Measurement of Adhesive Layer by Polarized Laser, Trans. Jpn. Soc. Mech. Eng., (in Japanese), Vol. 58, No. 554, A (1992), p. 1900.

(5) Niitsu, Y. and Ichinose, K., Stress Measurement of Transparent Solid Materials by Polarized Laser, Proc. of Int. Elec. Pack. Conf., Vol. 2,(1992), p. 1005.

(6) Niitsu, Y., Ichinose, K. and Ikegami, K., Stress Measurement of Transparent Materials by Polarized Laser, Trans. Jpn. Soc. Mech. Eng., (in Japanese), Vol. 59, No. 559, A (1993), p. 600.

( 7 ) Niitsu, Y., Ichinose, K. and Ikegami, K., A stress Measuring Method by Polarized Laser and Photo-Elastic Modulator, Proc. of Int. Elec. Pack. Conf., Vol. 1,(1993), p. 157.

(8) Ichinose, K. and Niitsu, Y., Scanning Stress Measurement Method by Laser Photoelasticity (1st Report, Stress Measurement by Synthesis Method), Trans. Jpn. Soc. Mech. Eng., (in Japanese), Vol. 60, No. 572, A(1994), p. 1114. 